\title{
Generation of special autosomal dominant polycystic kidney disease iPSCs with the capability of functional kidney-like cell differentiation
}

Jiahui Huang ${ }^{1,2,3}$, Shumin Zhou' ${ }^{1}$ Xin Niu' ${ }^{1}$ Bin Hu${ }^{1}$, Qing Li ${ }^{1}$, Feng Zhang ${ }^{4}$, Xue Zhang ${ }^{4}$, Xiujuan Cai ${ }^{5}$, Yuanlei Lou ${ }^{2}$, Fen Liu ${ }^{2}$, Chenming $\mathrm{Xu}^{6}$ and Yang Wang ${ }^{1 *}$

\begin{abstract}
Background: Human induced pluripotent stem cells (iPSCs) have been verified as a powerful cell model for the study of pathogenesis in hereditary disease. Autosomal dominant polycystic kidney disease (ADPKD) is caused by mutations of PKD or non-PKD genes. The pathogenesis of ADPKD remains unexplored because of the lack of a true human cell model.

Methods: Six ADPKD patients and four healthy individuals were recruited as donors of somatic cells from a Chinese ADPKD family without mutations of the PKD genes but carrying SAMSN1 gene deletion. The ADPKD-iPSCs were generated from somatic cells and were induced into kidney-like cells (KLCs) by a novel three-step method involving cytokines and renal epithelium growth medium. Furthermore, we analyzed functional properties of these KLCs by water transportation and albumin absorption assays.

Results: We successfully generated iPSCs from ADPKD patients and differentiated them into KLCs that showed morphological and functional characteristics of human kidney cells. Further, we also found that ADPKD-iPSC-KLCs had a significantly higher rate of apoptosis and a significantly lower capacity for water transportation and albumin absorption compared to healthy sibling-derived differentiated KLCs. Furthermore, knockdown of SAMSN1 in control iPSCs may attenuate differentiation and/or function of KLCs.

Conclusions: These data show that we have created the first iPSCs established from ADPKD patients without mutations in the PKD genes, and suggest that the deletion mutation of SAMSN1 might be involved in the differentiation and/or function of KLCs. ADPKD-iPSC-KLCs can be used as a versatile model system for the study of kidney disease.
\end{abstract}

Keywords: Induced pluripotent stem cells, Autosomal-dominant polycystic kidney disease, Differentiation, Kidney cells, SAMSN1

\section{Background}

Induced pluripotent stem cells (iPSCs), which were first reported by Yamanaka in 2006, are usually generated by reprogramming somatic cells by introducing a number of pluripotent factors, generally OCT4, SOX2, KLF4, C-MYC, LIN28 and NANOG [1-3]. iPSCs are characterized by an unlimited proliferative capacity and can be

\footnotetext{
*Correspondence: wangy63cn@126.com

${ }^{1}$ Institute of Microsurgery on Extremities, Shanghai Jiao Tong University Affiliated Sixth People's Hospital, Shanghai, People's Republic of China

Full list of author information is available at the end of the article
}

differentiated into the majority of cell types both in vivo and in vitro, offering an ideal tool for studying molecular and cellular mechanisms of hereditary diseases in vitro [4-7]. Autosomal dominant polycystic kidney disease (ADPKD) is a common life-threatening inherited renal disorder, characterized by the progressive formation of renal cysts and various extra-renal manifestations such as intracranial arterial aneurysms, and has a prevalence of approximately 1 in 400-1 in 1000 live births [8-11]. ADPKD results in severe destruction of normal renal parenchyma and eventually leads to renal failure. The 
majority of ADPKD patients ultimately enter end-stage renal disease (ESRD) in their 50s and 60s, and have to undergo dialysis therapy for the rest of their lives or receive kidney transplantation [12]. Genetic defects in two genes named PKD1 (polycystin-1; PC1) or PKD2 (polycystin-2; PC2) are associated with ADPKD. Mutations of these two PKD genes account for approximately $91 \%$ of the pathogenesis of the disease [13-15]. However, in approximately $9 \%$ of ADPKD cases mutations have not been detected [15-17]. In the absence of credible human cell models, the pathogenesis of ADPKD has not been investigated thoroughly. The construction of a cell model of ADPKD in vitro is an urgent task and is the key to discovering the pathogenesis of ADPKD.

In this study, we demonstrated the generation and characterization of iPSCs from ADPKD patients without PKD1/PKD2 mutations. These iPSCs are indistinguishable from human embryonic stem cells (hESCs) with respect to colony morphology, passaging, surface and pluripotent markers, normal karyotype, DNA methylation, and differentiation potential. We also describe and illustrate the efficient directed differentiation of ADPKDiPSCs into functional kidney-like cells (KLCs) in vitro; in addition, we reveal that low-level expression of the SAMSN1 gene can attenuate differentiation and function of KLCs in ADPKD. We are the first to establish iPSCs from ADPKD patients without mutations in the PKD1 or PKD2 genes, and our results show that a deletion mutation in the SAMSN1 gene might be involved in the differentiation and/or function of KLCs in ADPKD-iPSCs.

\section{Methods}

\section{Cell culture}

As shown in Fig. 1a, a Chinese ADPKD family containing ten living persons was selected for this study. The mother (LTP) and one of her sons (TSG) exhibited healthy phenotypes whereas the other three sons (TSB, TTB and THB) showed characteristic phenotypes of ADPKD. TSB exhibited the most severe, bilateral renal cysts of various sizes while THB had a relatively mild renal cyst phenomenon which was verified by ultrasound diagnosis (Fig. 1b). However, three daughters of THB (TLL, TII and TXM) exhibited ADPKD phenotypes while the other two grandchildren (TDS and TLY) of LTP exhibited healthy phenotypes. After ultrasound diagnosis, six members (TTB, TSB, THB, TLL, TII and TXM) exhibited a renal cyst phenomenon and were defined as "ADPKD patients", while the others (TSG, TLY, LTP and TDS) exhibited healthy phenotypes and were defined as "healthy persons". Human fibroblast cells (HFCs) and blood samples were obtained after the individuals provided informed consent; the consent forms are available upon request from the ethics committee of the First Affiliated Hospital of Nanchang University who approved the procedure. HFCs were maintained in fibroblast medium with Modified Eagle's medium/Nutrient Mixture F12 (DMEM/F12; Invitrogen, Rockville, MD, USA) supplemented with $10 \%$ fetal bovine serum (FBS; Hyclone, Logan, UT, USA) at $37{ }^{\circ} \mathrm{C}$. Blood samples were well preserved in liquid nitrogen.

\section{Gene mutation analysis \\ Sanger sequencing analysis}

A long-range PCR (LR-PCR) strategy followed by nested PCR was used for mutational analysis of the PKD1 gene. The duplicated region of PKD1 was amplified in eight specific long fragments by LR-PCR (exons $1,2-7,8-12$, 13-15, 15-21, 22, 23-28 and 29-34) [18] using TaKaRa LA Taq ${ }^{\text {Tu }}$ (TaKaRa Bio Inc.). Exons 1-34 of PKD1 were then amplified by nested PCR using these LR templates and exons 35-46 were directly PCR amplified and sequenced in both directions. The proband without pathogenic mutation in the $P K D 1$ gene was subsequently analyzed by mutational screening of the PKD2 gene via Sanger sequencing. Exons 1-15 of PKD2 including the adjacent $30-50 \mathrm{bp}$ intronic sequence were amplified from genomic DNA according to a method reported previously with little modification [19]. PCR amplification primers for the various LR-PCR fragments are presented in Table 1.

The pathogenicity of novel missense variants was evaluated computationally by SIFT, PolyPhen 2 and AlignGVGD prediction programs through analyzing interspecies sequence variations. When all programs classified it as damaging and no other definite mutation was found in the same patient, the variant was considered likely to be pathogenic. The variant would be regarded as a polymorphism in cases where all scores denied its pathogenicity. Otherwise, they were classified as "likely polymorphisms". Furthermore, pedigree cosegregation analysis of the potential pathogenic mutations in PKD2 was performed in all available members.

\section{Comparative genomic hybridization microarray}

Genomic DNA extracted from patient TSB together with sex-matched control DNA was fragmented by AluI and RsaI enzyme digestion. DNA labeling was conducted using an Agilent SureTag DNA Labeling Kit. Different fluorescence dyes were used for DNA labeling of patient DNA (Cy5-dUTP) and control DNA (Cy3-dUTP). The labeled products were mixed and hybridized onto Agilent SurePrint G3 human $1 \times \mathrm{v} 1 \mathrm{M}$ microarray for 40 hours at $65{ }^{\circ} \mathrm{C}$. DNA processing, microarray handling and scanning were conducted following the Agilent oligonucleotide comparative genomic hybridization (CGH) protocol (version 6.0). The microarray scanning profiles were processed by Agilent Feature Extraction 10.7.3.1. The extracted data were analyzed and plotted 


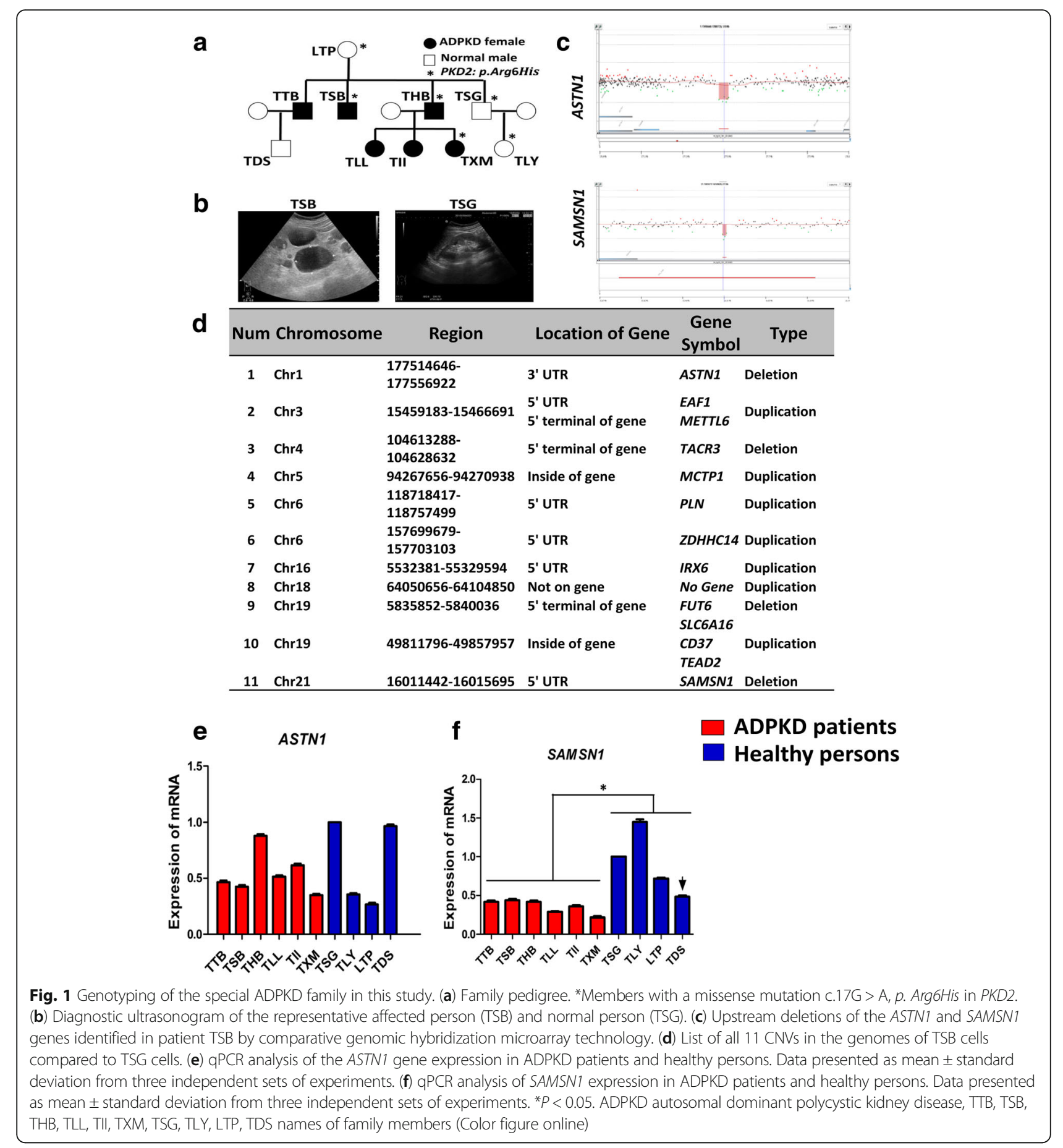

by Agilent Workbench 7.0. ADM-2 was selected as the statistical algorithm with the threshold set at 6.0 and Fuzzy Zero turned on.

\section{Generation of iPSCs}

The reprogramming process was developed as depicted diagrammatically in Additional file 1: Figure S1a. The key principle of the reprogramming approach is that the expression of four individual transcription factors
(OCT4, SOX2, KLF4 and $c-M Y C)$ and the expression of a cocktail of transcription factors (OCT4, SOX2, KLF4 and $c-M Y C)$ erases the active epigenetic network of a somatic cell and reopens a "ground state" of undifferentiated pluripotency $[1,2]$. We introduced the retroviruses containing human OCT4, SOX2, KLF4 and $c-M Y C$ produced in 293 T cells (PLAT-A packaging cells [20]) into HFCs (retroviral vectors obtained from Dr Duanqing Pei's laboratory, Guangzhou, China). HFCs from TSB, THB 
Table 1 Primers for PKD1 and PKD2

\begin{tabular}{|c|c|c|c|c|c|}
\hline Fragment & Size $(k b)$ & Exons & Forward primer & Reverse primer & Temperature $\left({ }^{\circ} \mathrm{C}\right)$ \\
\hline \multicolumn{6}{|l|}{ PKD1 } \\
\hline L1 & 2.2 & 1 & CCATCCACCTGCTGTGTGACCTGGTAAAT & CCACCTCATCGCCCCTTCCTAAGCAT & 68 \\
\hline L2 & 4.6 & $2-7$ & ATTाITGAGATGGAGCTTCACTCTTGCAGG & CGCTCGGCAGGCCCCTAACC & 68 \\
\hline L3 & 4.2 & $8-12$ & CCGCCCCCAGGAGCCTAGACG & CATCCTGTTCATCCGCTCCACGGTTAC & 68 \\
\hline$\llcorner 4$ & 4.4 & $13-15$ & TGGAGGGAGGGACGCCAATC & GTCAACGTGGGCCTCCAAGT & 68 \\
\hline L5 & 3.4 & $15-21$ & AGCGCAACTACTTGGAGGCCC & GCAGGGTGAGCAGGTGGGGCCATCCTA & 70 \\
\hline L6 & 0.3 & 22 & GAGGCTGTGGGGGTCCAGTCAAGTGG & AGGGAGGCAGAGGAAAGGGCCGAAC & 64 \\
\hline L7 & 4.2 & $23-28$ & CCCCGTCCTCCCCGTCCTTTTGTC & AAGCGCAAAAGGGCTGCGTCG & 68 \\
\hline L8 & 5.8 & $29-34$ & GGCCCTCCCTGCCTTCTAGGCG & GTTGCAGCCAAGCCCATGTTA & 68 \\
\hline $35-37$ & 0.7 & $35-37$ & GGGATGAATTCACAGCCTAC & GGAGACAAGAGACGGAGGT & 62 \\
\hline $38-40$ & 1.1 & $38-40$ & AAGCCCTGCTGTCACTGT & TACTCCCTTGTCCTTGGC & 56 \\
\hline $41-43$ & 1.1 & $41-43$ & GGGAGTAGTTCTCCAGGAGTG & CGAGAAATCTGTCTGCTTGC & 62 \\
\hline $44-46$ & 1.1 & $44-46$ & GGCTGCAAGCAGACAGATT & GCGGTGTCCACTCCGACTCC & 56 \\
\hline \multicolumn{6}{|l|}{ PKD2 } \\
\hline $1-1$ & 0.5 & 1 & AGAGGGAGGCGGGCCAAAGG & CGGGCGCCACTCTACGTCCA & 62 \\
\hline $1-2$ & 0.4 & 1 & GTGGAGCCGCGATAACCCCG & AGGCGGAACGCAGAGGGGAT & 62 \\
\hline 2 & 0.5 & 2 & TTGTGCTTTATTTCCCTITTGCCA & TGCCTCTCCCGTCCTGTGTT & 59 \\
\hline $3-4$ & 2.5 & $3-4$ & AGGGGAAAGGAAGGCAAGGGTGA & TGCCTTGGTGAAGGTGTCAGGGA & 65 \\
\hline $5-6$ & 4.0 & $5-6$ & GCCAGGTCAGGCACAGTACCC & AGCGTGGCTGAGAGCATACTGT & 63 \\
\hline $7-8$ & 4.5 & $7-8$ & TGGCAGGGCTTAACACTTTCCATTT & TCTTGAGAAGCAGTGACAACTCTGA & 65 \\
\hline $9-10$ & 4.7 & $9-10$ & ACCGTGCCCAGCTTGTGTTT & CTGCCGTGGAAGGTCAAGGG & 65 \\
\hline $11-13$ & 2.9 & $11-13$ & CCAGCACGTACTTGTTGAATGGCC & GGGAACTGCCTGGTCTCATGTGG & 65 \\
\hline $14-15$ & 1.0 & $14-15$ & GCCAGTGGGGCTGAAAAGACA & AGCATCCTATGGTGGTCAGGGCA & 70 \\
\hline
\end{tabular}

and TSG were used to reprogram into iPSCs. Approximately $4 \times 10^{4}$ HFCs were cultured until they reached $80 \%$ confluence and were transduced in fibroblast medium with a cocktail of retroviruses as reported previously [21, 22]. At day 2 post infection, medium was changed to DMEM/F12 containing 20\% FBS and $50 \mu \mathrm{g} / \mathrm{ml}$ Vitamin C (Vc) (sodium L-ascorbate; Sigma, St Louis, MO, USA). At day 6, infected HFCs were trypsinized and reseeded onto feeder cells (mitotically inactivated murine embryonic fibroblasts) and cultured in DMEM/F12 medium supplemented with $10 \% \mathrm{FBS}, 50 \mu \mathrm{g} / \mathrm{ml} \mathrm{Vc}, 1 \mathrm{mM} / \mathrm{ml}$ valproic acid (VPA; Merck, Darmstadt, Germany), 1 mM glutamax (Invitrogen), $0.1 \mathrm{mM}$ nonessential amino acids (NEAA; Invitrogen), $0.1 \mathrm{mM} \beta$-mercaptoethanol ( $\beta$-ME; Invitrogen) and $8 \mathrm{ng} / \mathrm{ml}$ basic fibroblast growth factor (bFGF; Shenzhen Symmix Industry, Shenzhen, China). VPA was added on days 7-22. Effectiveness of the cell transduction was assessed by the appearance of GFP-positive cells under fluorescent microscopy. Human ESC-like colonies appeared at around day 20 post infection and were picked up manually at around day 25. Picked iPSCs were routinely maintained on Matrigel $^{\circ}$ (Becton Dickinson, Franklin Lakes, NJ, USA) in mTeSR1 medium (Stemcell Technologies, Madison, WI, USA).

\section{Alkaline phosphatase staining}

The iPSCs were fixed with $4 \%$ paraformaldehyde (PFA) for 2 minutes at room temperature (RT) followed by two washes with sterile phosphate buffered saline (PBS), and then rinsed with Tris-buffered saline containing Tween20 (TBST; $20 \mathrm{mM}$ Tris-HCl, $\mathrm{pH} 7.4 ; 0.15 \mathrm{M} \mathrm{NaCl}$, $0.05 \%$ Tween-20). Freshly prepared alkaline phosphatase (AP) staining solution (Sidansai, Shanghai, China) was added to the colonies and they were incubated for 15 minutes at RT in the dark. The staining solution was then aspirated and the colonies were washed twice with sterile PBS and analyzed by light microscopy.

\section{Immunofluorescence staining}

The iPSCs were fixed in 4\% PFA for 20-30 minutes at RT, washed twice with sterile PBS, permeabilized in $0.5 \%$ Triton X-100 for 20 minutes and blocked with 5\% bovine serum albumin (BSA) solution in sterile PBS for 2 hours. The cells were then incubated with primary antibody in $0.5 \%$ BSA solution at $4{ }^{\circ} \mathrm{C}$ overnight. Next day, the primary antibody solution was aspirated, cells were washed five times for 30 minutes, and then incubated with the corresponding secondary antibody solution for 1 hour at $37^{\circ} \mathrm{C}$. Cells were washed, stained with 
4,6-diamino-2-phenylindole (DAPI; Sigma) for 5 minutes at RT, washed and covered in sterile PBS, and finally photographed under a fluorescence microscope (Leica Microsystems, Wetzlar, Germany).

For confocal imaging of primary cilia, cells were grown on glass coverslips up to day 29. The cells were fixed and acetylated alpha-tubulin immunofluorescence staining was performed as described previously [23]. Cells were imaged on a Zeiss LSM 5 Pascal confocal microscope (Carl Zeiss, Oberkochen, Germany) using a 1.4 numerical aperture in plane or a 2.8 numerical aperture in stack scan mode. Images were deconvolved using Zeiss LSM Examiner software (version 4.0.0.241). All antibodies used are presented in Table 2.

\section{In-vitro and in-vivo differentiation}

To test the differentiation capacity of iPSC lines, iPSC colonies growing on Matrigel $^{\circledR}$ were loosely detached by dispase treatment for 5 minutes, washed four times with DMEM/F12, scraped up with a glass pipette, and resuspended in DMEM/F12 medium containing 20\% knockout serum replacement (KSR; Gibco, Thermo Fisher Scientific, Waltham, MA, USA), $1 \mathrm{mM}$ glutamax, $0.1 \mathrm{mM}$ NEAA and $0.1 \mathrm{mM} \beta$-ME. Embryoid bodies (EBs) were maintained on $1 \%$ agar-coated lowattachment plates and replenished every 2 days with fresh EB medium (DMEM/F12 containing 20\% FBS). EBs were placed on Matrigel $^{\circ}$-coated plates after 8 days in suspension, and then allowed to differentiate for another 18 days in EB medium before processing for immunofluorescence analysis. As for teratoma formation, iPSCs were washed with DMEM/F12, treated with dispase for 5 minutes at $37{ }^{\circ} \mathrm{C}$, scraped up using a glass pipette, collected by centrifugation and resuspended in DMEM/F12 containing Matrigel ${ }^{\circ}$. Approximately $2 \times 10^{6}$ iPSCs were injected into immune-compromised NODSCID mice (Weitonglihua, Beijing, China). Eight weeks after injection, teratomas were dissected, rinsed once with sterile PBS, fixed with $10 \%$ formalin, embedded in paraffin and cut into sections 4-5 $\mu \mathrm{m}$ thick. Hematoxylin/eosin staining was performed as reported previously [24].

\section{Karyotype analyses}

The iPSCs were cultured in six-well plates until they reached $80-90 \%$ confluence before mitotic arrest was induced by treatment with $20 \mu \mathrm{g} / \mathrm{ml}$ colcemid for 2 hours. Following incubation, the colonies were digested using $0.25 \%$ trypsin ethylene diamine tetraacetic acid (EDTA) (Invitrogen), and cells were centrifuged at $2000 \times g$ for 5 minutes, resuspended in $7 \mathrm{ml}$ of $0.075 \mathrm{M} \mathrm{KCl}$, and incubated for 20 minutes at $37{ }^{\circ} \mathrm{C}$. Prefixative solution composed of one part acetic acid and three parts methanol was added, mixed gently, and incubated for 40 minutes at $37{ }^{\circ} \mathrm{C}$. After further centrifugation, the supernatant was removed. Cells were dropped onto a cold slide and incubated at $75^{\circ} \mathrm{C}$ for 3 hours. Giemsa banding was performed following a standard protocol with incubations in $0.05 \%$ trypsin for 8 seconds and Giemsa staining dilution for 10 minutes. Imaging and karyotyping were performed using Meta Systems Band View software.

\section{DNA fingerprinting using short tandem repeat analysis}

To confirm whether iPSCs were derived from ADPKD fibroblasts, short tandem repeat (STR) analysis was performed by the DNA Sequencing Core Facility at Technology Biological Co., Ltd, Shanghai Boyi.

Table 2 Primary antibodies

\begin{tabular}{|c|c|c|c|}
\hline Antibody & Isotype & Dilution & Source \\
\hline$\overline{\mathrm{OCT} 4}$ & Rabbit lgG & $1: 50$ & CST $2840 \mathrm{~s}$ \\
\hline SSEA-4 & Mouse lgG & $1: 50$ & Abcam 4755 \\
\hline TRA-1-60 & Mouse lgM & $1: 100$ & CST 4746 \\
\hline TRA-1-81 & Mouse IgM & $1: 50$ & CST 4745 \\
\hline SOX2 & Mouse lgG & $1: 100$ & CST $2748 \mathrm{~s}$ \\
\hline AFP & Mouse lgG & $1: 100$ & CST 3903 s \\
\hline Nestin & Rabbit lgG & $1: 200$ & Abcam ab105389 \\
\hline Bry & Rabbit lgG & $1: 200$ & Abcam ab20680 \\
\hline Desmin & Rabbit lgG & $1: 100$ & CST 5332 s \\
\hline Pax2 & Rabbit lgG & $1: 100$ & Abcam ab79389 \\
\hline AQP1 & Rabbit lgG & $1: 100$ & CST Sc52623 \\
\hline Blll-tubulin & Mouse lgG & $1: 200$ & CST $4466 \mathrm{~s}$ \\
\hline E-cad & Rabbit lgG & $1: 20$ & CST 3195 \\
\hline Synaptopodin & Mouse lgG & $1: 100$ & PROGEN Biotechnik SC6260 \\
\hline Anti-acetylated alpha tubulin & Mouse lgG & $1: 100$ & Abcam ab24610 \\
\hline
\end{tabular}




\section{Flow cytometry analyses}

The iPSCs were suspended with $0.05 \%$ trypsin for 10 minutes, centrifuged, and then resuspended in sterile PBS. The single cell suspension was fixed in 1\% PFA for 15 minutes at $37{ }^{\circ} \mathrm{C}$ and permeabilized in $90 \%$ precooled methanol for 30 minutes. Both primary and secondary antibody incubations were carried out according to the manufacturer's instructions. Control samples were stained with isotype-matched control antibodies. After washing, the cells were resuspended, filtered, and then used for flow cytometry (BD FACS Aria; Becton Dickinson). The antibodies used for flow cytometry are presented in Table 2 .

\section{Quantitative reverse transcription-polymerase chain reaction}

RNA was extracted using Trizol reagent (Invitrogen). qPCR was performed using an ABI7900 Thermal Cycler Dice ${ }^{\text {Th }}$ Real Time System (ABI, Foster City, CA, USA) and SYBR Green Premix EX Taq ${ }^{\text {tm }}$ (Takara, Shiga, Japan). GAPDH was used for normalization and all items were measured in triplicate. Quantitative reverse transcription-polymerase chain reaction (RT-qPCR) and semi-quantitative PCR primers are summarized in Table 3.

\section{Methylation analysis of gene promoters}

Bisulfite treatment was performed using a cytosine guanine dinucleotide $(\mathrm{CpG})$ modification kit (Promega, Madison, WI, USA) according to the manufacturer's recommendations. Amplified products were cloned into PCR2.1-TOPO (Takara). Twelve randomly selected clones were sequenced with the M13 forward and M13 reverse primers for each gene. PCR primers are presented in Tables 3 and 4.

\section{In-vitro kidney lineage differentiation studies}

The kidney differentiation capacity of human ADPKDiPSCs was developed as depicted diagrammatically in
Fig. 4a. To induce differentiation of KLCs from ADPKDiPSC colonies, colonies of H9 ESCs and ADPKD-iPSCs were cut into uniform-sized pieces, transferred into six-well plates precoated with Matrigel $^{\circ}$ for 1 hour, and cultured initially in mTeSR 1 medium until $30 \%$ confluent. To induce differentiation, mTeSR1 medium was replaced with stage 1 medium with DMEM/F12, containing 1\% Glutamax, 1\% NEAA and 10\% FBS, and supplemented with bone morphogenic protein 7 (BMP7), human vascular endothelial growth factor (hVEGF), bFGF and Activin-A (all from HumanZyme, Chicago, IL, USA) during days $1-3$ only (all at $10 \mathrm{ng} / \mathrm{ml}$ ) and with $5 \mu \mathrm{M}$ lithium for 14 days. To initiate differentiation toward the intermediate mesoderm (IM), cells were cultured for another 7 days with retinoic acid (RA; HumanZyme) supplemented with stage 1 medium without Activin-A. To induce renal precursor or mature cell differentiation, the cells were cultured with stage 1 medium in various combinations with renal epithelium growth medium (REGM; BioWhittaker, Walkersville, MD, USA) for another 7 days. The medium was changed every 2 days.

\section{Proliferation assay studies}

In-vitro cell proliferation assays were evaluated using a cell counting kit 8 (CCK8; Dojindo Molecular Technologies, Kumamoto, Japan) according to the manufacturer's instructions. The cells differentiated at days 21-28 were used for proliferation assays by CCK 8 reagent. Briefly, $720 \mu \mathrm{l}$ of fresh medium and $80 \mu \mathrm{l}$ of the CCK 8 solution were added to each well, and cells were incubated at $37^{\circ} \mathrm{C}$ for 1 hour. A blank well contained only the CCK8 reagent and medium without any cells. The absorbance at $450 \mathrm{~nm}$ was measured using an automatic microplate reader (BioTek Instruments, Winooski, VT, USA). All experiments were performed in triplicate.

Table 3 Primers for characterization

\begin{tabular}{|c|c|c|}
\hline Gene & Forward sequence & Reverse sequence \\
\hline ACTB & CCCAGAGCAAGAGAGG & GTCCAGACGCAGGATG \\
\hline endo-OCT4 & CCTCACTTCACTGCACTGTA & CAGGTITCTITCCCTAGCT \\
\hline endo-Sox2 & CCCAGCAGACTTCACATGT & СCTCCCATTTCCCTCGTTT \\
\hline Nanog & TGAACCTCAGCTACAAACAG & TGGTGGTAGGAAGAGTAAAG \\
\hline $\operatorname{Rex} 1$ & TCGCTGAGCTGAAACAAATG & СCCTTCTTGAAGGTITACAC \\
\hline AFP & ATTGGCAAAGCGAAGCTG & GCTGTGGCTGCCATITIT \\
\hline CK18 & AGCTCA ACGGGATCCTGCTGCACCTTG & CACTATCCGGCGGGGGTGGCTTTTG \\
\hline MSX1 & CACTATCCGGCGGGGGTGGCTITTG & CGAGAGGACCCCGTGGATGCAGAG \\
\hline TBX1 & AGCGAGAAATATGCCGAGG & TTCGCGAAGGGATTGCT \\
\hline PAX6 & TTCGCGAAGGGATTGCT & TGCCCGTTCAACATCCTT \\
\hline SOX1 & TITCCCCTCGCTTTCTCA & TGCAGGCTGAATTCGGTT \\
\hline PAX2 & AGATTCCCAGAGTGGTGTG & GGGTATGTCTGTGTGCCTGA \\
\hline
\end{tabular}


Table 4 Primers for kidney-like cell differentiation

\begin{tabular}{lll}
\hline Gene & Forward sequence & Reverse sequence \\
\hline BRY & GACTGCTTATCAGAACGAGG & TGTCAGAATAGGATTGGGAG \\
PAX2 & AACGACAGAACCCGACTATG & ATCCCACTGGGTCATTGGAG \\
AQP1 & ATTAACCCTGCTCGGTCCTT & ACCCTGGAGTTGATGTCGTC \\
E-cad & TCCCATGCCTACCTCACCTT & ACCCTGGAGTTGATGTCGTC \\
Synaptopodin & AGCCCAAGGTGACCCCGAAT & CCCTGTCACGAGGTGCTGGC \\
WT1 & GGACAGAAGGGCAGAGCAACCA & GTCTCAGATGCCGACCGTACAA \\
GADPH & GTCTCCTCTGACTTCAACAGCG & ACCACCCTGTTGCTGTAGCCAA \\
Lim1 & TCATGCAGGTGAAGCAGTTC & TCCAGGGAAGGCAAACTCTA \\
\hline
\end{tabular}

\section{Apoptosis studies}

For apoptosis analysis, both apoptotic and necrotic cells in kidney differentiation cultures were measured using the Annexin V FITC/propidium iodide (PI) apoptosis detection kit (Dojindo) following the manufacturer's protocol at 21-25 days. In brief, both adherent and floating cells were collected, $10^{6}$ cells were washed twice with cold sterile PBS and then resuspended in Annexin V-FITC binding buffer. FITC-conjugated Annexin V (100 $\mathrm{\mu l} /$ sample) was added and cells were incubated for 10 minutes at RT in the dark. Cells were then centrifuged and resuspended in binding buffer, and PI was added (100 $\mu \mathrm{l} /$ sample). Samples were kept on ice and incubated for 20 minutes in the dark. The total percentage of apoptotic cells was measured by counting the number of $\mathrm{FITC}^{+}$and $\mathrm{FITC}^{+} / \mathrm{PI}^{+}$stained cells by Guava easyCyte6HT ${ }^{\mathrm{rm}}$ flow cytometry (5000 events/gate). Representative data from one of three independent experiments were analyzed using its built-in INCYTE (version 2.7) software (EMD Millipore, Merck).

\section{Water transport assay}

Cells cultured in differentiation medium for 28 days and the human kidney (HK2) positive cells were rinsed with PBS and loaded with CFSE (Invitrogen) for 10 minutes. After washing with sterile PBS, cells were incubated in a hypotonic solution $(0.06 \% \mathrm{NaCl}$ in water). The fluorescence intensity in the supernatant was then measured by Synergy $^{\mathrm{TM}}$ HT (Bio-Tek).

\section{Cell permeability}

A cell permeability assay was used to determine the endocytic uptake of fluorescence-labeled albumin as further evidence of glomerulus-like or podocyte-like functional characteristics. The protocol was as reported previously [25]. iPSCs differentiated for 28 days and primary podocyte-positive cells were cultured in serum-free medium with/without rhodamine-labeled albumin (0.5 mg/ml; Abcam, Cambridge, MA, USA) at $37{ }^{\circ} \mathrm{C}$ for 1 hour, while control cells were cultured at $4{ }^{\circ} \mathrm{C}$. After washing with sterile PBS, the cells were fixed in 4\% PFA and counterstained with DAPI solution. Finally the cells were photographed under a fluorescence microscope (Leica microscopy) and the fluorescence intensity was measured by Image J 1.48 .

\section{Knock down of the SAMSN1 gene}

The short hairpin RNAs (shRNAs) presented in Table 5 were used to target SAMSN1. ShSAMSN1 and negative control shRNA were synthesized and inserted into a lentivirus shuttle vector containing an enhanced green fluorescent protein $(E G F P)$ reporter gene and a puromycin antibiotic resistance gene. Expression of the shRNA was driven by the $\mathrm{H} 1$ promoter. Recombinant lentiviruses expressing SAMSN1-shRNA or negative control shRNA ( $L v$-shSAMSN1 and Lv-shNC) were produced in $293 \mathrm{~T}$ cells. TSG-iPSCs were infected with concentrated $L v$ shSAMSN1 or Lv-shNC virus in serum-free medium. The supernatant was replaced with complete culture medium after 6 hours. The stable knockdown cells were established by selection in complete culture medium containing puromycin $(1.0 \mu \mathrm{g} / \mathrm{ml})$ for approximately 14 days and then validated by qRT-PCR analysis. All of the primers are presented in Table 5.

\section{Statistical analysis}

All assays were performed in triplicate. Results are reported as the mean $\pm \mathrm{SD}$, and statistical significance was displayed as $P<0.05$ and $P<0.01$. Significant differences between two groups were determined by the independent Student's $t$ test.

Table 5 Primers for SAMSN1 knockdown

\begin{tabular}{ll}
\hline Name & Sequence $\left(5^{\prime} \rightarrow 3^{\prime}\right)$ \\
\hline shRNA-391 & GGAGAGAATGCCCACCCATAT \\
shRNA-601 & GCCAGAGTGCATACGGATTC \\
shRNA-707 & GGACAGGAATGTTGAACAATA \\
shRNA-840 & GGAGTTCCTAGAGAGGATTCA \\
shNC & GTTCTCCGAACGTGTCACGT \\
\hline
\end{tabular}




\section{Results}

\section{Genotyping of the special ADPKD family}

We first collected blood samples from all ten members of this family, including TSB (patient) and TSG (healthy sibling). Then we sequenced the PKD1 and PKD2 genes in patient TSB and his healthy brother TSG by Sanger sequencing. The proband (patient TSB) without pathogenic mutation in the PKD1 gene was subsequently analyzed by mutational screening of the PKD2 gene via Sanger sequencing. A novel missense mutation c.17G > A, p. Arg6His in PKD2 was found in the proband. As shown in Fig. 1a, however, this mutation was also found in his healthy relatives (LTP, TSG and TLY), and was found to be absent from other affected family members (TTB, TLL and TII); thus the variant did not segregate with the disease in the family. According to our prediction standard, the mutation in PKD2 c.17G > A, p. Arg6His was therefore predicted to be a polymorphism (Additional file 2: Figure S2).

We then hypothesized that other changes in the genome might play a key role in this special ADPKD family. TSB and TSG were analyzed by CGH microarrays. Eleven genes' copy number variation (CNV) regions were detected (Fig. 1d), including deletions of ASTN1 and SAMSN1 mutations (Fig. 1c). However, no apparent deletion or duplication mutations were found at the loci of PKD1 and PKD2 genes (Additional file 3: Figure S3a). We then validated all CNV regions using RT-qPCR. Nine of eleven did not pass the verification and CNVs containing the $5^{\prime}$ upstream sequence of genes ASTN1 and SAMSN1 were consistent with the phenotypes of TSB and TSG (Additional file 3: Figure S3b). We further confirmed these two CNVs by RT-qPCR in all of the family members (Fig. 1e, f). Only CNV containing the 5' upstream sequence of gene SAMSN1 corresponded to the phenotype of individuals in this family (Fig. 1f) with the exception of TDS (Fig. 1f, arrowhead). So far, TDS did not exhibit renal cyst phenomenon by ultrasound diagnosis, while the SAMSN1 gene expression in TDS displayed a relatively low level like ADPKD patients. Because the ADPKD patients usually take a long time to become symptomatic and TDS is only a 15 -year-old boy, we inferred that TDS might exhibit a renal cyst phenomenon at an older age.

We can conclude from these two experiments that PKD mutations can be ruled out in this ADPKD family. However, CNV of the SAMSN1 gene was identified as a possible candidate and consequently we would then proceed to validation analysis.

\section{Characterization of ADPKD-iPSCs}

Six iPSC clones from these individuals (TSB, THB and TSG) were generated in our study, and three iPSC clones (TSG iPSCs, THB iPSCs and TSB iPSCs) were selected for further characterization. To detect the expression of pluripotency markers in the picked colonies, iPSCs that were positive for AP activity (Additional file 1: Figure S1b) were analyzed by immunostaining and flow cytometry (FCM) for OCT4, SSEA4, TRA-1-60 and TRA1-81, and the results showed that all four pluripotency marker proteins were upregulated (Fig. 2a). To further confirm that the selected colonies were really iPSCs, exogenous and endogenous pluripotency marker genes were analyzed by semi-quantitative PCR and qPCR in the genomic DNA of iPSCs. As shown in Fig. 2b, all integration exogenous pluripotency genes were overexpressed in all iPSCs compared to those in fibroblasts on day 6 . Nineteen days later, the integration exogenous transcription factors were silenced in all iPSCs by host cells (Fig. 2c), whereas endogenous pluripotency factors were activated in all iPSCs (Fig. 2d), which proved that the selected colonies had been reprogrammed successfully. H1 ESCs were positive cells.

In order to analyze whether the genome of established iPSCs was stable and normal, karyotype analysis was performed as described previously [26]. As shown in Fig. 2e, both iPSC lines (TSG, TSB and THB) displayed a normal karyotype of 46XY (data for THB not shown). We then studied the methylation status of CpG in the promoter regions of key transcription factors by bisulfite sequencing, and found that the promoters of OCT4 and NANOG displayed extensive DNA demethylation in iPSCs, in contrast to their parent fibroblasts (Fig. 2f). In addition, genetic STR analysis confirmed that the iPSCs were derived from this individual's fibroblasts and were not contaminated with other cell lines grown in our laboratory (Fig. 2g). All of the characterization results of the three iPSC lines show a similar tendency.

\section{In-vitro and in-vivo differentiation of ADPKD-iPSCs}

To further confirm the pluripotency of these special ADPKD-iPSCs, in-vitro and in-vivo differentiation capability were performed as described. For in-vitro EB differentiation, ADPKD-iPSC gobbets were maintained in suspension culture for EB formation for about 8 days followed by 18 days in attachment culture in order to differentiate into EBs (Fig. 3a, light view); protein markers of three germ layers were then analyzed by fluorescent immunostaining. As shown in Fig. 3a, the expression and localization of marker proteins representing endoderm cells ( $\alpha$-fetoprotein; AFP), mesoderm cells (Desmin, Brachyury: BRY), and ectoderm cells $\left(\beta_{\mathrm{III}^{-}}\right.$ tubulin, Nestin) could be clearly detected, which demonstrated the pluripotency of ADPKD-iPSCs. Furthermore, qPCR analyses of more extensive marker genes of the three germ layers and pluripotency markers were also performed to confirm EB differentiation. As expected, the expressions of differentiation markers (AFP, CK18, 


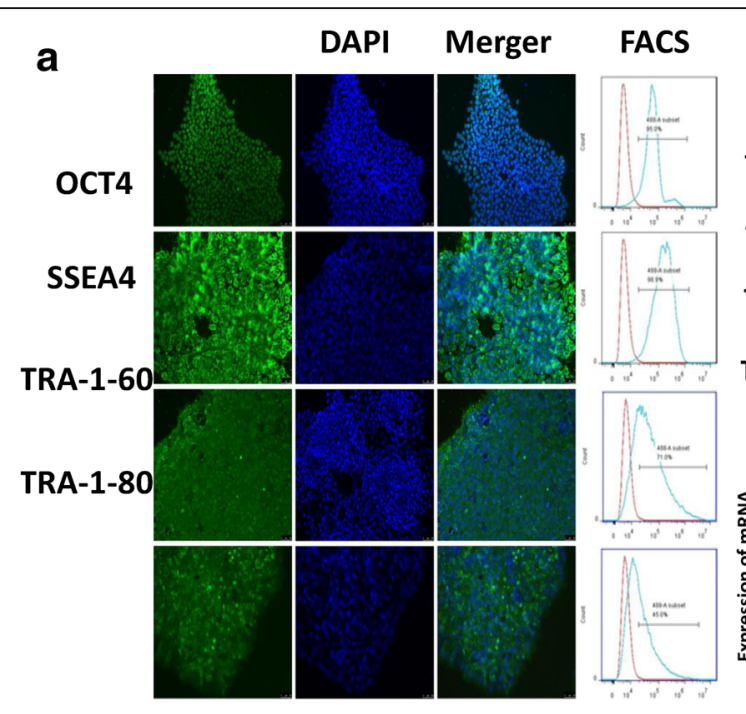

b

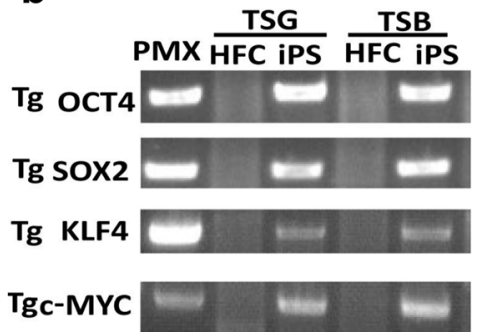

C

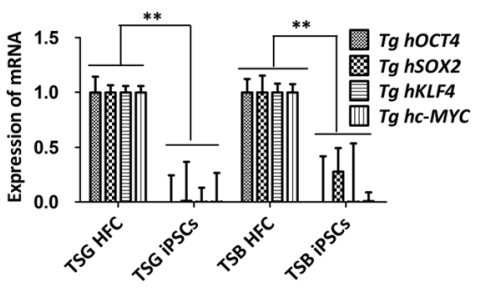

d

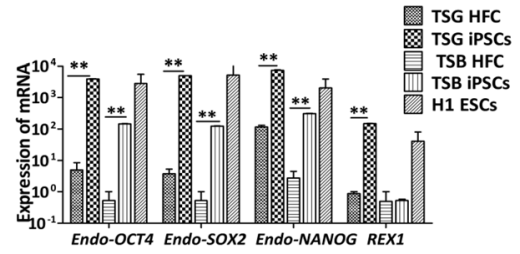

e TSG iPSCs (Healthy)

TSB iPSCS (ADPKD)

If If if II I) if II is II II

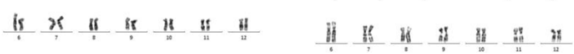

"

f

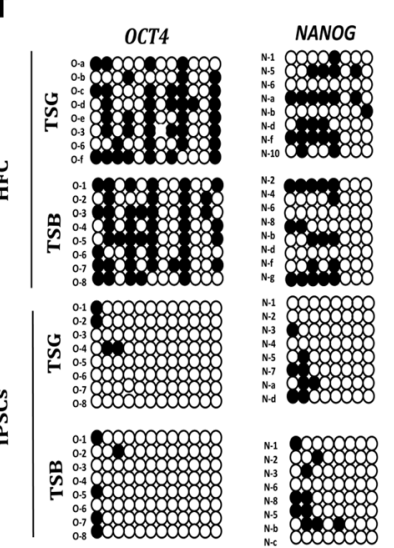

g

\begin{tabular}{|l|l|l|l|l|l|l|l|l|}
\hline $\begin{array}{l}\text { Locus/Sa } \\
\text {-mple }\end{array}$ & \multicolumn{2}{|l|}{ TSG iPSCS } & \multicolumn{2}{|l|}{ TSG HFC } & \multicolumn{2}{|l|}{ TSB iPSCS } & \multicolumn{2}{|l}{ TSB HFC } \\
\hline D75820 & 7.8 & 11 & 7.8 & 11 & 11 & & 11 & \\
\hline CSF1PO & 9.6 & 11.6 & 9.6 & 11.6 & 9.6 & 11.6 & 9.6 & 11.6 \\
\hline TH01 & 8.8 & & 8.8 & & 8.8 & & 8.8 & \\
\hline D13S317 & 10.3 & 11.3 & 10.3 & 11.3 & 10.3 & 11.3 & 10.3 & 11.3 \\
\hline D165539 & 8.9 & & 8.9 & & 8.9 & & 8.9 & \\
\hline vWA & 14.2 & 18.4 & 14.2 & 18.4 & 14.2 & 18.4 & 14.2 & 18.4 \\
\hline TPOX & 8.2 & 9.2 & 8.2 & 9.2 & 8.2 & & 8.2 & \\
\hline D55818 & 6.6 & 9.7 & 6.6 & 9.7 & 9.7 & 12.8 & 9.7 & 12.8 \\
\hline $\begin{array}{l}\text { Ameloge- } \\
\text { in }\end{array}$ & $\mathrm{X}$ & $\mathrm{Y}$ & $\mathrm{X}$ & $\mathrm{Y}$ & $\mathrm{X}$ & $\mathrm{Y}$ & $\mathrm{X}$ & $\mathrm{Y}$ \\
\hline
\end{tabular}

Fig. 2 Generation and characterization of ADPKD-iPSCs. (a) Immunofluorescence staining and FCM analysis of ADPKD-iPSC colonies. Expression of iPSC specific proteins (OCT4, SSEA4, TRA-1-60 and TRA-1-81) (first column) with corresponding DAPI-stained nuclei (second column) and merged images (third column). These cells were also analyzed by FCM and positive rates were tested. Bar $=50 \mu \mathrm{m}$. (b) Semi-quantitative PCR results showing that expressions of exogenous genes were overregulated in iPSCs after day 6 during programming. (c) qPCR showing that expressions of exogenous genes in iPSCs were silent after day 19 during programming. Data presented as mean \pm standard deviation from three independent sets of experiments. ${ }^{*} P<0.01$. (d) qPCR results showing upregulated expression of endogenous iPSC specific genes in healthy or ADPKD-iPSCs. Human embryonic stem cells (H1 ESCs) acted as a positive control. Data presented as mean \pm standard deviation from three independent sets of experiments. ${ }^{*} P<0.01$. (e) ADPKD specific iPSC colonies showing a normal 46XY karyotype. (f) Methylation status of eight CpGs analyzed (one per row) in the promoter region of both OCT4 and NANOG genes from twelve or eight randomly sequenced clones represented as $8 \times$ 12 and $8 \times 8$ matrices, respectively, for both iPSCs and human fibroblast cells (HFCs). Open circles indicate the unmethylated state and dark, filled circles indicate the methylated state, which overall indicated that the loci tested are highly methylated in HFCs, while they have been reprogrammed to the unmethylated state in the iPSC colonies. (g) Genomic fingerprint analysis of TSG and TSB in both PSSCs and their corresponding HFCs. ADPKD autosomal dominant polycystic kidney disease, ESC embryonic stem cell, iPSC induced pluripotent stem cell, TSB, TSG names of family members (Color figure online) 
a
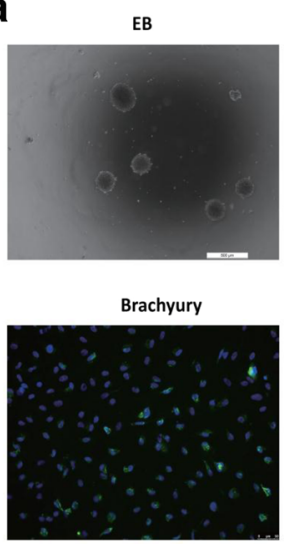

Nestin

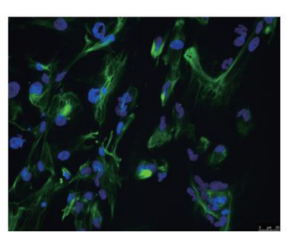

b

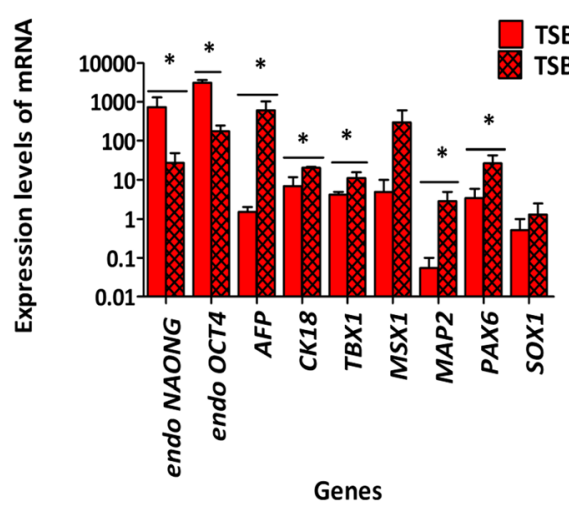

d

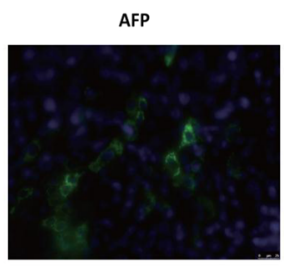

Desmin

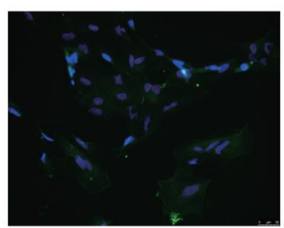

$\beta$-Tubulin
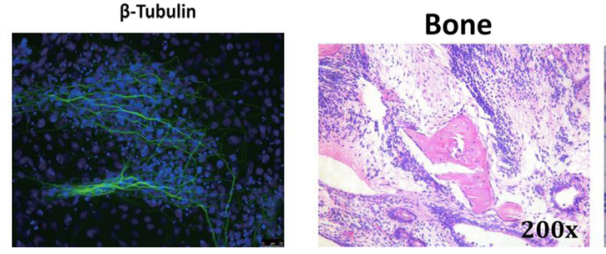

Glandular tissue

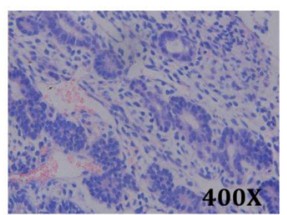

Neural roettes

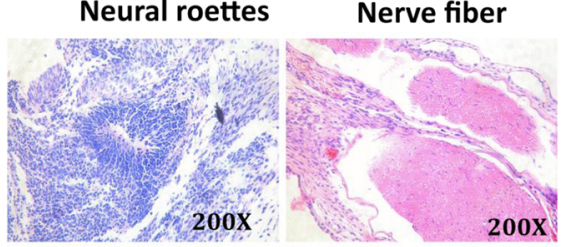

Cattilage

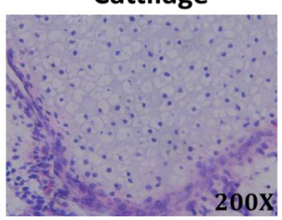

C

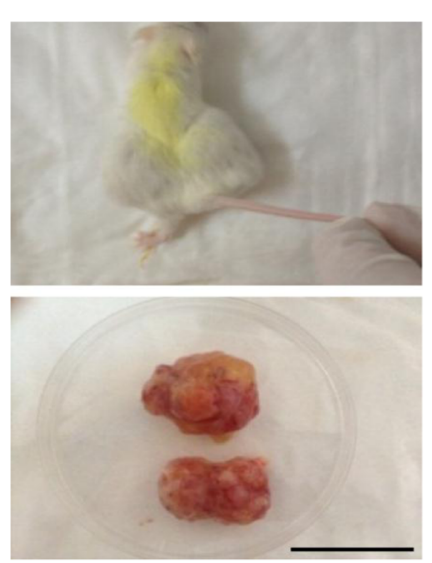

Fig. 3 In-vitro and in-vivo differentiation of ADPKD-iPSCs. (a) Embryoid body (EB) formation by ADPKD-specific iPSCs in suspension culture. Differentiated EBs expressed markers from all three germ layers, including a-fetoprotein (AFP; endoderm, bar $=25 \mu \mathrm{m}$ ), Nestin and Desmin (mesoderm), Brachyury: BRY and $\beta_{\| 11}$ tubulin (ectoderm). Bar $=50 \mu \mathrm{m}$. (b) qPCR analysis showing differences in gene expression patterns between undifferentiated iPSCS and differentiated EBs. Undifferentiated iPSCs expressed high levels of endogenous OCT4 and NANOG genes while EBs expressed high levels of marker genes of all three layers. Data presented as mean \pm standard deviation from three independent sets of experiments. ${ }^{*} P<0.05$. (c) Teratomas evident following the injection of undifferentiated ADPKD-specific iPSCs into immunodeficient mice. Bar $=1 \mathrm{~cm}$. (d) Hematoxylin and eosin staining of tissues from all three germ layers. Bar $=1 \mathrm{~cm}$. ADPKD autosomal dominant polycystic kidney disease, iPS induced pluripotent stem cell, TSB name of family member (Color figure online)

TBX1, MSX1, MAP1, SOX1 and PAX6) in EB differentiated cells were increased compared to that of in ADPKDiPSCs while the expressions of pluripotency markers (endo-NANOG and endo-OCT4) were decreased (Fig. 3b).

In addition, the pluripotent properties of these iPSCs were assessed by teratoma formation in vivo. NODSCID mouse recipients were employed for differentiation by iPSCs injection. The formation of teratomas (Fig. 3c) was observed (3/3 mice), which produced derivatives of the three germ layers including rather complex structures in the case of teratomas (Fig. 3c). Taken together, our analyses of ADPKD-iPSCs derived from retrovirally transduced ADPKD patient fibroblasts confirmed their pluripotent potential.

\section{Directed differentiation of ADPKD-iPSCs into KLCs}

In order to model the progress of ADPKD in vitro, it is necessary to first induce iPSCs to differentiate into the kidney lineage cells. The stepwise differentiation method we set up simulated the process of kidney generation in 
embryo development through three main phases: mesoderm, intermediate mesoderm (IM) and KLCs. As depicted diagrammatically in Fig. 4a, iPSCs were induced in ABVF (Activin-A, BMP7, hVEGF and bFGF) condition medium for at least 28 days by adding lithium chloride, retinoic acid (RA) and REGM. At 28 days, examination of the differentiated ADPKD-iSPCs showed that they had developed into two morphologically different cell types. One was large, often multinucleated and arborized cells with cytoplasmic extensions (Fig. 4b, lower left). The morphology was comparable to conditionally immortalized human podocytes (Fig. 4b, upper left) [27]. The other type consisted of fusiform and fibroblast-like cells (Fig. 4b, lower right) which appeared similar to human kidney (HK2) cells under the light microscope (Fig. 4b, upper right). We also performed immunofluorescence staining of primary cilium, which is a surface feature of podocytes (Additional file 1: Figure S1c, red arrow), and found no difference in cilium formation between ADPKD-iPSCs and normal iPSCs.

To map the process of differentiation of ADPKDiPSCs into KLCs, markers of genes corresponding to the three phases were analyzed by qPCR. The results showed that the expression of genes characteristic of

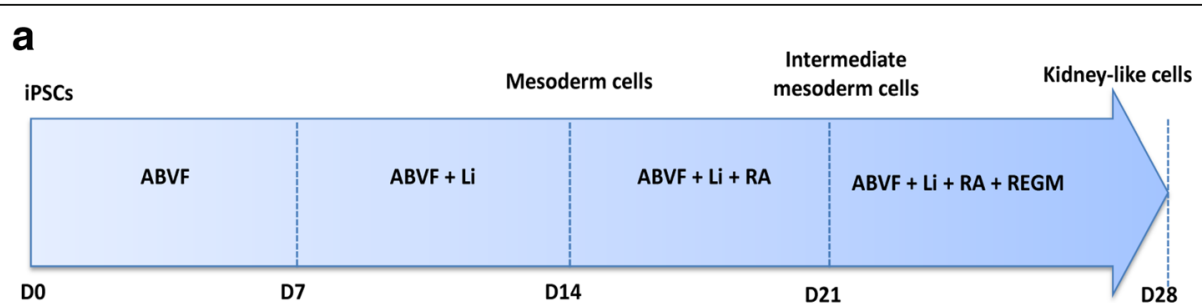

b

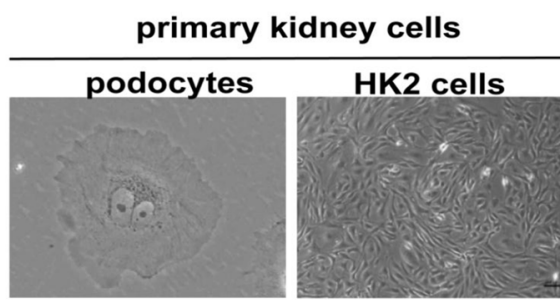

iPSCs induced KLCs

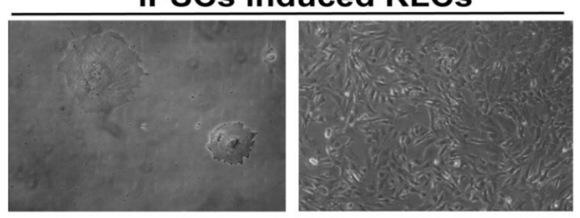

C

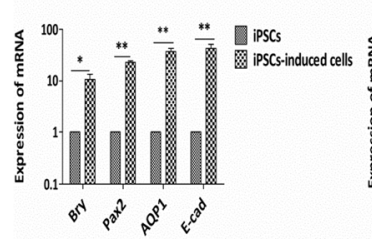

d

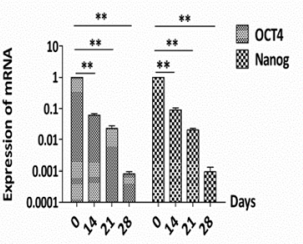

e
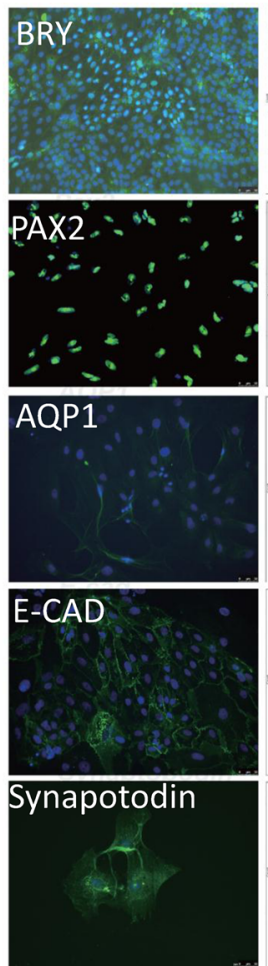

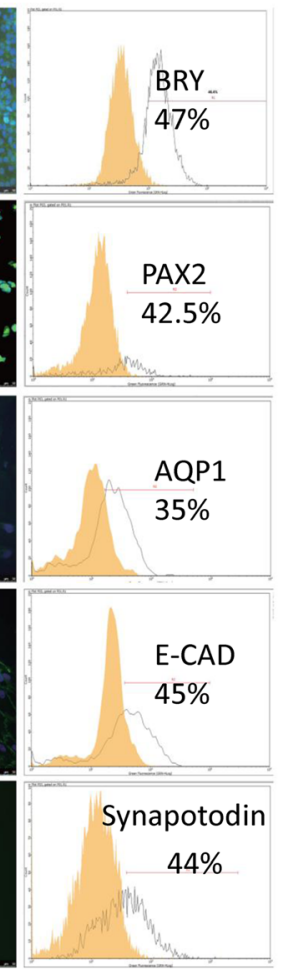

Fig. 4 Direct differentiation of ADPKD-iPSCs into kidney-like cells (KLCs). (a) Scheme showing the stepwise protocol used for producing KLCs from ADPKD-iPSCs and the time needed. (b) Morphology of induced ADPKD-iPSCs is similar to podocytes and human kidney (HK2) cells. Bar = 100 $\mu$ m. (c) Upregulation of marker genes of each stage during differentiation from iPSCs into functional KLCs. Values (mean of three replicates) are referred to the undifferentiated iPSCs. Data presented as mean \pm standard deviation from three independent sets of experiments, ${ }^{*} P<0.05$, ${ }^{* *} P<0.01$. (b) Pluripotency of iPSCs decreased during induction to KLCs. Data are averages and standard deviations of three independent experiments. Values (mean of three replicates) are referred to the undifferentiated iPSCs. ${ }^{*} P<0.01$. (e) Immunofluorescence and FCM results of marker genes of each step of induction. BRY is a marker of mesoderm cells; PAX2 a marker for intermesoderm cells; and synaptopodin, AQP1, and E-cadherin (E-CAD) are markers for KLCs. Bar $=50 \mu \mathrm{m}$. iPSC induced pluripotent stem cell, RA retinoic acid, REGM renal epithelium growth medium, ABVF ActivinA, BMP7, hVEGF and bFGF 
mesoderm, IM and KLCs were increased (Fig. 4c) whereas those of the pluripotency genes (OCT4 and NANOG) were decreased (Fig. 4d) during kidney lineage cell differentiation compared to iPSCs. Human fetal kidney cDNA was used as positive control. Furthermore, the proportions of $\mathrm{BRY}^{+}$cells (47\%), $\mathrm{PAX}^{+}$cells (42.5\%), $\mathrm{AQP}^{+}$cells $(35 \%), \mathrm{E}-$ cadherin $^{+}$cells $(45 \%)$ and synaptopodin $^{+}$cells (44\%) were obtained from FCM assay (Fig. 4e, right), and immunofluorescence staining also confirmed these results (Fig. 4e, left).

\section{Differentiated iPSCs from ADPKD patients and healthy individuals expressed different phenotypes}

Because the ADPKD-iPSCs could be induced to differentiate into KLCs in the same way as the healthy human iPSCs or H9 ESCs, we investigated whether the phenotypes of the differentiated KLCs derived from ADPKDiPSCs were different from those derived from healthy human iPSCs. During the induction process, no obvious differences in cell morphology were observed based on light microscopy (data not shown). However, the cell proliferation capacity was different by the CCK8 assay. The proliferation viabilities of ADPKD-iPSC differentiated cells dropped continuously compared to those of TSG iPSC differentiated cells at days 21-26 (Fig. 5a).

Previous studies have reported that increased apoptosis is an early event in ADPKD [28], therefore we analyzed the apoptosis rates of these two iPSC line differentiated cells by double labeling with Annexin V-FITC/propidium iodide (PI) followed by FCM. Early apoptotic cells were stained with Annexin V-FITC whereas late apoptotic cells and necrotic cells were stained with both Annexin V-FITC and PI. As shown in Fig. 5b, these two iPSC lines after differentiation for 21 days showed a similar percentage of cells undergoing early apoptosis (Annexin V-FITC staining only; 41.33\% vs $41.71 \%)$. However, the percentage undergoing early apoptosis (Fig. 5b, orange bar) in ADPKD at day 23 was increased compared to the healthy cells (purple bar) $(46.2 \%$ vs $30.12 \%)$, and the percentage undergoing late apoptosis in ADPKD at day 25 was significantly increased compared to the control cells ( $52.7 \%$ vs $28.88 \%$ ). The results confirmed that the apoptosis rate of cells differentiated from ADPKD-iPSCs was higher than that from healthy iPSCs at days 21-25 of the induction.

Water transport ability is generally used to estimate kidney tubule function [29]. Using an improved version of the reported protocol [29], we measured the water transport ability of differentiated KLCs by measuring the fluorescent product of CFSE washed out under hypotonic conditions at 2-minute intervals. In this case, differentiated KLCs and HK2 cells both showed a similar response under hypotonic conditions (Additional file 1: Figure S1d). Surprisingly, the assay results also showed that the water transportation function of KLCs was much weaker in TSB iPSCs than in TSG iPSCs (Fig. 5c). HK2 cells were used as positive control. To estimate functional characteristics of KLCs, a cell permeability assay was used to determine the endocytic uptake of albumin as further evidence of glomerulus and podocytelike functional characteristics [25]. After 28 days of differentiation, rhodamine-albumin was employed to detect the albumin absorption function of KLCs differentiated from these two iPSC lines. The intensity of red fluorescence decreased by $76 \%(P<0.01)$ in TSB iPSC-KLCs compared to that of in TSG iPSC-KLCs (Fig. 5e-g).

To further investigate whether the expression level of SAMSN1 changes in the process of kidney cell differentiation were consistent with the genomic changes between ADPKD patients and healthy individuals, we then performed qPCR for SAMSN1 as well as other kidneyrelated genes (Fig. 5d). Expression levels were varied among the different genes but in general exhibited the same expression tendency in ADPKD-iPSCs and healthy iPSCs (Fig. 5d). SAMSN1 mRNA levels in the ADPKD patient were lower than in the healthy individual almost throughout the induction period. Meanwhile the $A Q P 1$ values were significantly lower in differentiated ADPKDiPSCs than in healthy iPSCs (Fig. 5d), which might explain why comparable results were obtained in the water transport assay.

\section{Knockdown of SAMSN1 may attenuate differentiation and/or function of KLCs in ADPKD}

We hypothesized that deletion of the $5^{\prime}$ UTR of SAMSN1 may reduce its expression and in turn attenuate differentiation or function of KLCs in ADPKD. TSG iPSCs infected with blank virus were used as control (Fig. 6a), and a stable TSG iPSC SAMSN1 cell line infected with shSAMSN1 virus was established using a lentivirus system (Fig. 6b). Using the qPCR assay, we found that the SAMSN1 expression level was knocked down by $35 \%(P<0.01)$ in TSG SAMSN1 cells compared to control TSG iPSCs after puromycin selection for about 14 days (Fig. 6c). No apparent morphological differences were observed between them (data not shown). After kidney cell differentiation for 7 days, the SAMSN1 expression level in TSG SAMSN1 cells was decreased to below $40 \%(P<0.01)$ compared to TSG control cells (Fig. 6c).

Next, we aimed to verify whether the biological behaviors of KLCs derived from TSG SAMSN1 iPSCs was the same as that of ADPKD-iPSCs. Both TSG control iPSCs and TSG SAMSN1 iPSCs underwent kidney differentiation at the same time. After 28 days of differentiation, rhodamine-albumin was also employed to analyze the albumin absorption function of these two induced iPSCs. The intensity of red fluorescence was decreased by $63 \%$ 


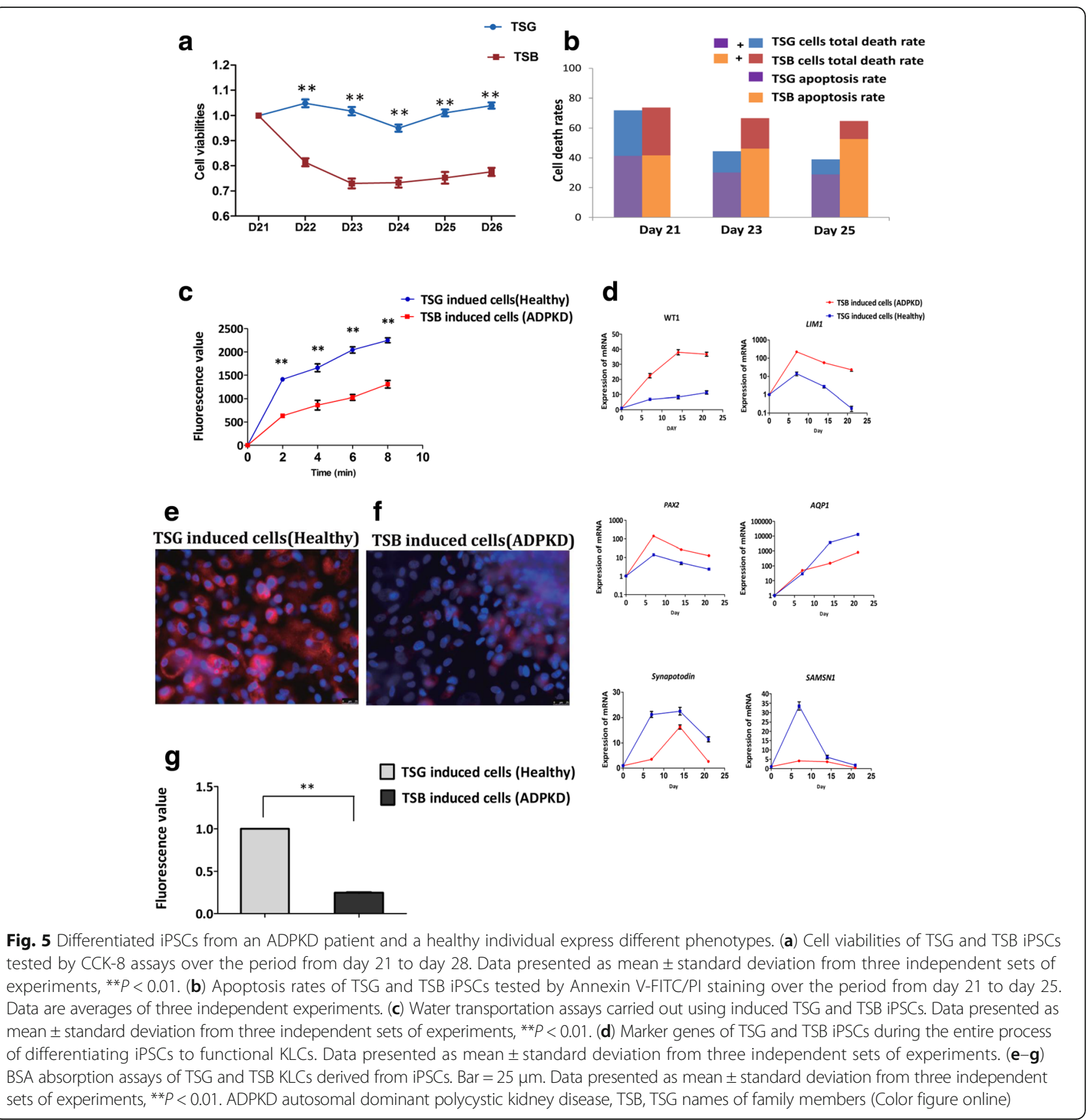

$(P<0.01)$ in TSG SAMSN1-induced iPSCs compared to that of in TSG control iPSCs (Fig. 6d-f). Moreover, the water transportation assay was also performed. TSG SAMSN1-induced iPSCs also had a slightly reduced water transport ability compared to those of TSG control cells (Fig. 6g). This indicates that the functionality of KLCs in the TSG SAMSN1-induced iPSCs was reduced compared to TSG control iPSCs. These two functional results were consistent with the earlier results (Fig. 5c, e-g). All of these results verified that some biological behaviors of KLCs derived from TSG SAMSN1 iPSCs were changed like ADPKD compared to TSG control iPSCs. The deletion of the 5' UTR of SAMSN1 reduced its expression and may attenuate the differentiation or function of KLCs in ADPKD.

\section{Discussion}

Although decades have passed since the discovery of PKD1/PKD2 mutations in ADPKD, the pathogenesis of ADPKD remains unexplored and it remains unclear which other genes contribute to the pathogen of ADPKD. To personalize the study of the unique pathology of ADPKD, we first established and characterized ADPKD-iPSCs from a special ADPKD family without defects in the PKD1/ 


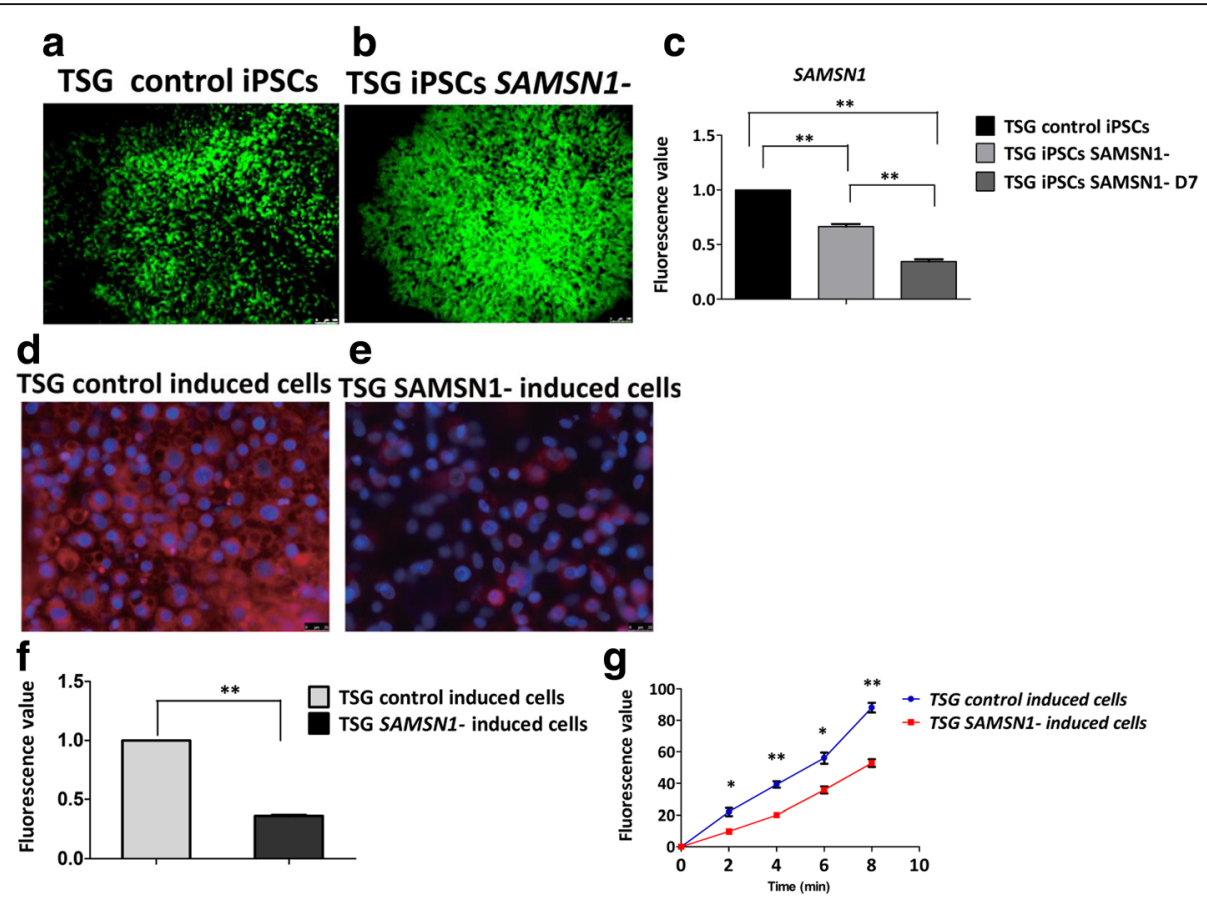

Fig. 6 Knockdown of SAMSN1 may attenuate differentiation and/or function of KLCs in ADPKD. (a-c) Morphology of TSG control induced cells and TSG SAMSN1-induced iPSCS and the relative expression rates of SAMSN1 in TSG SAMSN1-induced iPSCS compared to those in TSG control-induced cells. Bar $=100 \mu \mathrm{m}$. Data presented as mean \pm standard deviation from three independent sets of experiments, ${ }^{* *} P<0.01$. (d-f) BSA absorption assays of TSG control-induced cells and TSG SAMSN1-induced iPSCs. Bar $=25 \mu \mathrm{m}$. Data presented as mean \pm standard deviation from three independent sets of experiments, ${ }^{*} P<0.01$. (g) Results of water transportation assays of TSG control-induced cells and TSG SAMSN1-induced iPSCs. Data presented as mean \pm standard deviation from three independent sets of experiments, ${ }^{*} P<0.05$, ${ }^{*} P<0.01$. ADPKD autosomal dominant polycystic kidney disease, iPSC induced pluripotent stem cell, TSG name of family member (Color figure online)

PKD2 genes. We also reported a novel method of inducing human iPSCs to differentiate into functional KLCs, and the differentiated KLCs derived from ADPKD or his healthy sibling had different phenotypes and functions. Further, we found a rare mutation in the $5^{\prime}$ UTR of the SAMSN1 gene, which may attenuate KLCs differentiation or/and function in ADPKD.

The use of iPSCs for disease modeling is based on the fact that these cells are capable of self-renewal and can be differentiated into all types of cells of the human body, and can therefore be utilized for the preparation of different disease models to study disease pathogenesis. Moreover, disease-specific iPSCs could be of enormous use as far as development of specific therapeutic regimens/drugs is concerned. For example, this technique has been used to generate motor neurons from iPSCs of a patient with spinal muscular atrophy (SMA) that showed selective deficits compared to those derived from the child's unaffected mother [6]. This was the first study to demonstrate that human iPSCs can be used to model the specific pathology in a genetically inherited disease. Subsequently, more and more reports have shown that iPSCs derived from specific diseases provide good models for disease [22, 30-32]. In the case of ADPKD, although pathogenesis was studied previously, pathogenesis remained undetermined mostly because of the pathogenic gene polymorphisms or the existence of a third pathogenic gene [16]. ADPKD-iPSCs have previously been generated successfully but the genotypes were rarely described [33] or involved $P K D$ gene mutations [34, 35]. In our study, ADPKD-iPSC lines have been generated from a Chinese ADPKD family without PKD1 or PKD2 gene mutations. The special ADPKD-iPSCs are so different from other ADPKD-iPSCs reported previously that this could provide a new opportunity for the study of ADPKD pathogenesis.

There are no widely accepted protocols for directed differentiation of human iPSCs into kidney epithelial cells that give rise to kidney cysts; although ADPKDiPSCs have been established and induced to differentiate into hepatoblasts/epithelial cells [34] and vascular cells [35], the differentiation capacity of kidney cells has not yet been investigated. In our study, we combined the currently existing protocols of differentiating stem cells into IM cells and established a new stepwise protocol for inducing ADPKD-iPSCs to differentiate into KLCs (Fig. 4a). From the morphological observations, we concluded that the differentiated KLCs contain two kinds of cell populations which were similar to human podocytes and HK2 cells (Fig. 4b). We found that expression levels 
of mesoderm gene $B R Y$ and kidney cell differentiation genes were significantly increased compared to corresponding ADPKD-iPSCs in these two populations of cells during kidney differentiation (Fig. 4c). Meanwhile, the expression levels of NANOG and OCT4 were downregulated (Fig. 4d), and immunofluorescence and FCM analysis confirmed these results (Fig. 4d, e). In addition, the differentiated KLCs were positive for acetylated alpha-tubulin, marking the primary cilia protein from confocal image stacks (Additional file 1: Figure S1c). Three-dimensional projections of these stacks demonstrated primary cilia extending beyond the cell surface, as reported previously [35-37]. A recent study reported that differentiated cells from human ESCs displayed water transport functional characteristics of human primary renal proximal tubular cells [29]. We consulted and modified Narayanan et al.'s protocol [29]. SCFE, instead of calcein-AM, was employed to detect the cumulative fluorescence intensity using Synergy ${ }^{\text {Tax }}$ HT (Bio-Tek), and we found that differentiated KLCs and HK2 cells both showed a similar response tendency (Additional file 1: Figure S1d). Meanwhile, Song et al. [38] reported that the differentiated podocytes displayed podocyte-like functional characteristics by detecting the uptake of FITClabeled albumin. Using this protocol, we also observed absorption of rhodamine-albumin in the cytoplasm of the differentiated cells (Fig. 5e, f). The primary podocytes were used as positive control cells (Additional file 1: Figure S1e). It was surprising to find that the ADPKDiPSC (TSB) differentiated KLCs showed a downregulation in these two functional experiments compared to healthy controls (TSG) (Fig. 5f, g). For these experiments, we used KLCs differentiated from H9 ESCs, two ADPKD-iPSC clones (TSB and THB) and a normal iPSC clone (TSG). We present the results of patient TSB and healthy sibling TSG because these similar results have existed in two ADPKD-iPSCs. Taken together, these data indicate for the first time that ADPKD-iPSCs could be induced to differentiate into functional kidney cells but with lower functional characteristics compared to healthy control iPSCs.

As reported formerly, SAMSN1 (also known as HACS1/ $N A S H 1 / S L Y 2)$ is widely expressed in hematopoietic tissues, muscle, heart, brain, lung, pancreas, endothelial cells and myelomas, usually acts as an immunoinhibitory adaptor, and plays significant roles in the development and regulation of immunocytes such as B cells, T cells and mast cells in both mouse and human [39]. In our gene mutation study, we found that the ADPKD patients did not have any $P K D 1$ or $P K D 2$ gene mutations but did present a deletion in the 5' UTR of the SAMSN1 gene (Fig. 1c lower panel, d). Further, the SAMSN1 gene expression levels of all ADPKD patients were lower than those of other healthy family persons (Fig. 1f). Besides this, a recent study revealed that SAMSN1 induces Rac1-dependent membrane ruffle formation and regulates cell spreading and polarization by reorganizing the cytoskeletal actin network, which counteracts excessive B-cell spreading [40]. Podocytes also have polygonal epithelial morphology and large cytoplasmic extensions [27], which demand extensive remodeling of the actin network. In our case, the KLC populations have polarized polygonal epithelial morphology and large cytoplasmic extensions that might be induced or regulated by SAMSN1. Consequently we hypothesized that the defect in the 5' UTR of the SAMSN1 gene reduced the expression of SAMSN1, which in turn affected KLC differentiation or function in ADPKD. To test this we first knocked down the SAMSN1 expression in TSG-iPSCs (TSG SAMSN1 iPSCs) and then induced the TSG SAMSN1 iPSCs to differentiate into KLCs, and found that SAMSN1 expression was significantly decreased by approximately $70 \%$ at day 7 after KLC differentiation (Fig. 6c). Next, we tested the water transportation and albumin absorbing functions, and in these two assays these differentiated KLCs derived from TSG SAMSN1 iPSCs both showed a downregulated tendency, which indicated that fewer functional KLCs and/or weaker functional KLCs were generated after SAMSN1 knockdown. So we thought that SAMSN1 may affect KLCs differentiation and/or function in ADPKD development. Taken together, our data suggest that the 5' UTR deletion of SAMSN1 may affect KLCs differentiation and/or function and provide a meaningful hint for the occurrence and development of ADPKD. Finally, there is a deficiency in this study that includes ten persons' samples from one family. In order to further clarify the significance of SAMSN1 mutation in ADPKD, we will continue to expand the sample size to verify the results of this article.

\section{Conclusions}

These results revealed that special ADPKD-iPSCs without PKD1/PKD2 gene mutations can be generated and induced to differentiate into functional KLCs using our modified differentiation protocol. We also show that the deletion mutation in SAMSN1 might be involved in KLCs differentiation and/or function in ADPKD and thus provide a new perspective to illustrate the underlying mechanism in ADPKD. We believe that ADPKDiPSC-KLCs hold huge potential to be used as versatile model systems for the study of kidney disease.

\section{Additional files}

Additional file 1: Figure S1. The additional characterization analysis for ADPKD-iPSC and KLCs. (a): The timeline and culture conditions of induction of fibroblasts to iPSCs. Lower panel; phase contrast microscopy showing each of the three major steps. Bar =100um. (b): AP staining for stemness of stem cells in iPSC lines. Bar $=100 \mathrm{um}$. (c): Immunofluorescence photomicrographs showing primary cilia (arrow head) in KCLs were generated from iPSCs. Bar $=5 \mathrm{um}$. (d): Water transportation assays were 
carried out between HK2 positive cells and KCLs. Data are represented as mean \pm standard deviation from three independent sets of experiments. (e): The podocyte was used as a positive control and absorbed rhodamine-albumin. Bar =25um. (JPG $1230 \mathrm{~kb}$ )

Additional file 2: Figure S2. The Sanger sequencing analysis for $P K D$ in a Chinese ADPKD family. (a): The novel missense mutation c.17 G > A, p.Arg6His in PKD2 was predicted by three program. (b): The list of all ten persons analyzed for the mutations. (c): The real sequencing pictures of all ten individuals in this family. (JPG $4280 \mathrm{~kb}$ )

Additional file 3: Figure S3. The comparative genomic hybridization (CGH) microarray analysis for PKD in a Chinese ADPKD family. (a): Representative image of CGH analyses of the PKD1 and PKD2 genes in patient TSB and healthy TSG. (b): QPCR verification of all eleven variants detected by CGH microarray in patient TSB and healthy TSG. Shown are the averages of three independent experiments. (JPG $3730 \mathrm{~kb}$ )

Additional file 4: Ethical approval file. (JPG $45 \mathrm{~kb}$ )

\section{Abbreviations}

ADPKD: Autosomal dominant polycystic kidney disease; ALP/AP: Alkaline phosphatase; bFGF: Basic fibroblast growth factor; BMP7: Bone morphogenic protein 7; BSA: Bovine serum albumin; CCK8: Cell counting kit 8; CNV: Copy number variation; DAPI: 4,6-Diamino-2-phenyl indole; DMEM/F12: Dulbecco's Modified Eagle's medium/Nutrient Mixture F12; EBs: Embryoid bodies; EDTA: Ethylene diamine tetraacetic acid; EGFP: Enhanced green fluorescent protein; ESC: Embryonic stem cell; ESRD: End-stage renal disease; FBS: Fetal bovine serum; FCM: Flow cytometry; HK2: Human kidney 2; hVEGF: Human vascular endothelial growth factor; IM: Intermediate mesoderm; iPSC: Induced pluripotent stem cell; KLC: Kidney-like cell; KSR: Knockout serum replacement; LR-PCR: Long-range PCR; NEAA: Nonessential amino acids; PFA: Paraformaldehyde; PI: Propidium iodide; PKD1: Polycystin-1; PKD2: Polycystin-2; RA: Retinoic acid; REGM: Renal epithelium growth medium; SMA: Spinal muscular atrophy; STR: Short tandem repeat; TBST: Tris-buffered saline containing Tween-20; Vc: Vitamin C; VPA: Valproic acid; ß-ME: $\beta$-Mercaptoethanol

\section{Acknowledgements}

The authors would like to thank Dr Duanqing Pei at Chinese Academy of Sciences for providing assistance in ADPKD-iPSC reprogramming.

\section{Funding}

This work was supported by the National Nature Science Foundation Grants (81170640, 30960385) and the National S\&T Major Special Project on Major New Drug Innovation (2011ZX09102-010-01).

\section{Availability of data and materials}

All data generated or analyzed during this study are included in this published article and its supplementary information files.

\section{Authors' contributions}

YLL and FL were responsible for skin and blood sample collection. $\mathrm{JH}, \mathrm{XN}, \mathrm{BH}$, $\mathrm{QL}$, and XJC participated in the experiments of cell and animal experiments. FZ and $X Z$ participated in the experiments of CNVs and gene analysis. CMX was responsible for gene mutation analysis in PKD1 and PKD2. JHH and SMZ contributed to data analysis and manuscript writing. YW conceived the idea, designed the experiments, and provided administrative support and final approval of manuscript. All authors read and approved the final manuscript.

\section{Ethics approval and consent to participate}

All procedures of experiments were approved by the Ethics Committee of Nanchang University Affiliated first Hospital (Additional file 4: Ethical approval). Written informed consent was obtained from all donors.

\section{Consent for publication}

Not applicable.

\section{Competing interests}

The authors declare that they have no competing interests.

\section{Publisher's Note}

Springer Nature remains neutral with regard to jurisdictional claims in published maps and institutional affiliations.

\section{Author details}

${ }^{1}$ Institute of Microsurgery on Extremities, Shanghai Jiao Tong University Affiliated Sixth People's Hospital, Shanghai, People's Republic of China. ${ }^{2}$ Institute of Urology First Affiliated Hospital of Nanchang University, Nanchang, People's Republic of China. ${ }^{3}$ Department of Clinical Laboratory, Sixth Affiliated Hospital of Sun Yat-Sen University, Guangzhou, People's Republic of China. ${ }^{4}$ State Key Laboratory of Genetic Engineering, School of Life Sciences, Fudan University, Shanghai, People's Republic of China. ${ }^{5}$ Key Laboratory of Regenerative Biology, South China Institute for Stem Cell Biology and Regenerative Medicine, Guangzhou Institutes of Biomedicine and Health, Chinese Academy of Sciences, Guangzhou, People's Republic of China. ${ }^{6}$ Institute of Embryo-Fetal Original Adult Disease Affiliated to Shanghai Jiao Tong, University School of Medicine, Shanghai, People's Republic of China.

Received: 12 July 2016 Revised: 16 July 2017

Accepted: 14 August 2017 Published online: 19 September 2017

\section{References}

1. Takahashi K, Yamanaka S. Induction of pluripotent stem cells from mouse embryonic and adult fibroblast cultures by defined factors. Cell. 2006;126(4):663-76.

2. Takahashi K, Tanabe K, Ohnuki M, Narita M, Ichisaka T, Tomoda K, et al. Induction of pluripotent stem cells from adult human fibroblasts by defined factors. Cell. 2007:131(5):861-72.

3. Yu J, Vodyanik MA, Smuga-Otto K, Antosiewicz-Bourget J, Frane JL, Tian S, et al. Induced pluripotent stem cell lines derived from human somatic cells. Science. 2007;318(5858):1917-20.

4. Walker FO. Huntington's disease. Lancet. 2007:369(9557):218-28.

5. Carvajal-Vergara X, Sevilla A, D'Souza SL, Ang Y-S, Schaniel C, Lee D-F, et al. Patient-specific induced pluripotent stem-cell-derived models of LEOPARD syndrome. Nature. 2010;465(7299):808-12.

6. Ebert $A D, Y u$ J, Rose FF, Mattis VB, Lorson $C L$, Thomson JA, et al. Induced pluripotent stem cells from a spinal muscular atrophy patient. Nature. 2009; 457(7227):277-80.

7. Marchetto MC, Carromeu C, Acab A, Yu D, Yeo GW, Mu Y, et al. A model for neural development and treatment of Rett syndrome using human induced pluripotent stem cells. Cell. 2010;143(4):527-39.

8. Dalgaard OZ. Bilateral polycystic disease of the kidneys; a follow-up of 284 patients and their families. Dan Med Bull. 1957;4(4):128-33.

9. Iglesias CG, Torres VE, Offord KP, Holley KE, Beard CM, Kurland LT. Epidemiology of adult polycystic kidney disease, Olmsted County, Minnesota: 1935-1980. Am J Kidney Dis. 1983;2(6):630-9.

10. Peters DJ, Sandkuijl LA. Genetic heterogeneity of polycystic kidney disease in Europe. Contrib Nephrol. 1992;97:128-39.

11. Torres VE, Harris PC. Autosomal dominant polycystic kidney disease: the last 3 years. Kidney Int. 2009;76(2):149-68.

12. Braun WE. Autosomal dominant polycystic kidney disease: emerging concepts of pathogenesis and new treatments. Cleve Clin J Med. 2009;76(2):97-104.

13. Tan Y-C, Blumenfeld J, Rennert H. Autosomal dominant polycystic kidney disease: genetics, mutations and microRNAs. Biochim Biophys Acta. 2011; 1812(10):1202-12.

14. Rossetti S, Consugar MB, Chapman AB, Torres VE, Guay-Woodford LM, Grantham JJ, et al. Comprehensive molecular diagnostics in autosomal dominant polycystic kidney disease. J Am Soc Nephrol. 2007;18(7):2143-60.

15. Audrezet MP, Cornec-Le Gall E, Chen JM, Redon S, Quere I, Creff J, et al. Autosomal dominant polycystic kidney disease: comprehensive mutation analysis of PKD1 and PKD2 in 700 unrelated patients. Hum Mutat. 2012; 33(8):1239-50

16. Ariza M, Alvarez V, Marin R, Aguado S, Lopez-Larrea C, Alvarez J, et al. A family with a milder form of adult dominant polycystic kidney disease not linked to the PKD1 (16p) or PKD2 (4q) genes. J Med Genet. 1997;34(7):587-9.

17. Yu C, Yang Y, Zou L, Hu Z, Li J, Liu Y, et al. Identification of novel mutations in Chinese Hans with autosomal dominant polycystic kidney disease. BMC Medical Genetics. 2011;12(1). 
18. Phakdeekitcharoen B, Watnick TJ, Germino GG. Mutation analysis of the entire replicated portion of PKD1 using genomic DNA samples. J Am Soc Nephrol. 2001;12(5):955-63.

19. Tan YC, Blumenfeld JD, Anghel R, Donahue S, Belenkaya R, Balina M, et al. Novel method for genomic analysis of PKD1 and PKD2 mutations in autosomal dominant polycystic kidney disease. Hum Mutat. 2009;30(2):264-73.

20. Morita S, Kojima T, Kitamura T. Plat-E: an efficient and stable system for transient packaging of retroviruses. Gene Ther. 2000;7(12):1063-6.

21. Zhang S, Chen S, Li W, Guo X, Zhao P, Xu J, et al. Rescue of ATP7B function in hepatocyte-like cells from Wilson's disease induced pluripotent stem cells using gene therapy or the chaperone drug curcumin. Hum Mol Genet. 2011;20(16):3176-87.

22. Lee G, Papapetrou EP, Kim H, Chambers SM, Tomishima MJ, Fasano CA, et al. Modelling pathogenesis and treatment of familial dysautonomia using patient-specific iPSCs. Nature. 2009:461(7262):402-6.

23. Kotsis F, Nitschke R, Doerken M, Walz G, Kuehn EW. Flow modulates centriole movements in tubular epithelial cells. Pflugers Arch. 2008;456(6):1025-35.

24. Prelle K, Zink N, Wolf E. Pluripotent stem cells-model of embryonic development, tool for gene targeting, and basis of cell therapy. Anat Histol Embryol. 2002;31(3):169-86.

25. Rastaldi MP, Song B, Smink AM, Jones CV, Callaghan JM, Firth SD, et al. The directed differentiation of human iPS cells into kidney podocytes. PLoS One. 2012;7(9):e46453.

26. Navarro S, Moleiro V, Molina-Estevez FJ, Lozano ML, Chinchon R, Almarza E, et al. Generation of iPSCs from genetically corrected Brca2 hypomorphic cells: implications in cell reprogramming and stem cell therapy. Stem Cells. 2014;32(2):436-46.

27. Saleem MA, O'Hare MJ, Reiser J, Coward RJ, Inward CD, Farren T, et al. A conditionally immortalized human podocyte cell line demonstrating nephrin and podocin expression. J Am Soc Nephrol. 2002;13(3):630-8.

28. Ibrahim S. Increased apoptosis and proliferative capacity are early events in cyst formation in autosomal-dominant, polycystic kidney disease. Sci World J. 2007;7:1757-67.

29. Narayanan K, Schumacher KM, Tasnim F, Kandasamy K, Schumacher A, Ni M, et al. Human embryonic stem cells differentiate into functional renal proximal tubular-like cells. Kidney Int. 2013;83(4):593-603.

30. Ku S, Soragni E, Campau E, Thomas EA, Altun G, Laurent LC, et al. Friedreich's ataxia induced pluripotent stem cells model intergenerational GAATTC triplet repeat instability. Cell Stem Cell. 2010;7(5):631-7.

31. Moretti $A$, Bellin $M$, Welling $A$, Jung CB, Lam JT, Bott-Flugel $L$, et al. Patientspecific induced pluripotent stem-cell models for long-QT syndrome. N Engl J Med. 2010;363(15):1397-409.

32. Singh VK, Kalsan M, Kumar N, Saini A, Chandra R. Induced pluripotent stem cells: applications in regenerative medicine, disease modeling, and drug discovery. Front Cell Dev Biol. 2015;3(2).

33. Thatava T, Armstrong AS, De Lamo J, Edukulla R, Khan Y, Sakuma T, et al. Successful disease-specific induced pluripotent stem cell generation from patients with kidney transplantation. Stem Cell Res Ther. 2011;2(6):48.

34. Freedman BS, Lam AQ, Sundsbak JL, latrino R, Su X, Koon SJ, et al. Reduced ciliary polycystin-2 in induced pluripotent stem cells from polycystic kidney disease patients with PKD1 mutations. J Am Soc Nephrol. 2013;24(10):1571-86.

35. Ameku T, Taura D, Sone M, Numata T, Nakamura M, Shiota F, et al. Identification of MMP1 as a novel risk factor for intracranial aneurysms in ADPKD using iPSC models. Sci Rep. 2016;6:30013.

36. Malone AMD, Anderson CT, Tummala P, Kwon RY, Johnston TR, Stearns $\mathrm{T}$, et al. Primary cilia mediate mechanosensing in bone cells by a calcium-independent mechanism. Proc Natl Acad Sci U S A. 2007;104(33):13325-30.

37. Yoder BK. Role of primary cilia in the pathogenesis of polycystic kidney disease. J Am Soc Nephrol. 2007;18(5):1381-8.

38. Song B, Smink AM, Jones CV, Callaghan JM, Firth SD, Bernard CA, et al. The directed differentiation of human iPS cells into kidney podocytes. PLoS One. 2012;7(9):28

39. Brandt S, Ellwanger K, Beuter-Gunia C, Schuster M, Hausser A, Schmitz I, et al. SLy2 targets the nuclear SAP30/HDAC1 complex. Int J Biochem Cell Biol. 2010;42(9):1472-81.

40. von Holleben M, Gohla A, Janssen KP, Iritani BM, Beer-Hammer S. Immunoinhibitory adapter protein Src homology domain 3 lymphocyte protein 2 (SLy2) regulates actin dynamics and B cell spreading. J Biol Chem. 2011;286(15):13489-501.

\section{Submit your next manuscript to BioMed Central and we will help you at every step:}

- We accept pre-submission inquiries

- Our selector tool helps you to find the most relevant journal

- We provide round the clock customer support

- Convenient online submission

- Thorough peer review

- Inclusion in PubMed and all major indexing services

- Maximum visibility for your research

Submit your manuscript at www.biomedcentral.com/submit

) Biomed Central 